\title{
Study on the Coupling Development between Urbanization and Ecosystem-- The Comparative Analysis Based on Guizhou, Yunnan, Hunan and Zhejiang Province
}

\author{
Lingyun Luo ${ }^{1, *}$,Chenggang $\mathrm{Li}^{1}$,Jue $\mathrm{Hu}^{2}$,Bing Yang $^{1}$ and Kang $\mathrm{Pan}^{1}$ \\ ${ }^{1}$ Faculty of Finance, Guizhou University of Finance and Economics, Guiyang, China \\ ${ }^{2}$ College of Textiles, Donghua University, Shanghai, China \\ Corresponding Email: 1ly0089@163.com
}

\begin{abstract}
Urbanization and ecological environment coordinated development is the development direction of contemporary urbanization. In this paper, the coupling degree model is used to analyze the coupling degree and coupling coordination degree of Guizhou, Yunnan, Hunan and Zhejiang Province in 2014. The analysis results showed that, four provinces are highly coupled in the coupling degree. Guizhou province is in the low degree of disorder and the lag of urbanization for the coupling coordination degree; Yunnan province is also in a low degree of disorder and urbanization hysteresis; Zhejiang province is in a good coordination, while its urbanization is slightly lagging behind; Hunan province is in a low degree of imbalance, and there is a coordinated development of urbanization and ecosystem. In this paper, the research results help four provinces above to better realize the coordinated development of urbanization and ecological environment.
\end{abstract}

\section{Introduction}

Urbanization refers to the historical transformation process from the primary industry-based agricultural society to the second and third industry-oriented modern urbanization of one country or region during the development of economy. Nowadays China is vigorously promoting the development of urbanization. As domestic demand is the fundamental driving force of China's economic development, and urbanization helps in expanding the domestic demand. Therefore, urbanization can be a strong engine to keep the sustained and healthy development of the economy. At present, China's urbanization rate of resident population is $53.7 \%$, and the urbanization rate of household registration population is only about $36 \%$, which is $80 \%$ lower than that of the developed countries. Guizhou Province as a western province, the urbanization rate is far behind the national average urbanization value. The urbanization rate in western China is $44.8 \%$, while the urbanization rate in Guizhou is only $42 \%$. In addition, the distribution of urbanization is unbalanced. As a result of this phenomenon, the urbanization potential of Guizhou is quite huge. So urbanization is regarded as the main strategy for Guizhou's development $[1,2]$. From the past experience, the development of urbanization will inevitably bring about the destruction of ecological environment. Over the past few years, China has only focused on the develop of economy, while do not pay any attention to the protection of the ecological environment. Thus it leads to enormous environmental pollution. In recent years, our country decided to take the road of sustainable development, which means, when we focus on the development of economy, we should pay attention to the protection of the ecological environment at the same time. In this paper, the coupling model is used to analyze the relationship between urbanization and ecological environment in order to promote the coordinated development of urbanization and ecological environment.

\section{Literature review}

Domestic and foreign scholars have carried out a series of researches on the coordinated development of urbanization and ecological environment. By the panel regression empirical study, Grossman (1995)found that the relationship between the ecological environment and the economic development was inverted U-shaped. In the early stage, because of the economic development, people ignored the protection of the environment. After a certain stage, people began to pay attention to environmental protection and governance, and then put forward the famous environmental Kuznets Curve [3]. Haughton $G$ et al. (1994) considered that the coupling relationship between urbanization and ecological environment is the sum of all kinds of non-linear relations between the elements of urbanization and the elements of ecological environment [4]. Domestic experts and scholars also carried on a certain research on the coupling model and the urbanization. Yan-ling Xiao (2013) used coupling model to calculate the coupling degree of urbanization and 
ecological environment coordinated development in Guizhou Province from 2001 to 2010, and obtained the conclusion that the coupling degree of urbanization and ecological environment coordinated development in Guizhou is increasing, In 2010, the coupling degree has reached 0.9545 [5]. Neng-sheng Luo et al. (2014) calculated the coupling degree of urbanization and environmental coordinated development in three cities of Changsha, Zhuzhou and Xiangtan. It was concluded that the coupling degree of Changsha was higher, from 0.293 in 2001 to 0.758 in 2011. However, Zhuzhou and Xiangtan City's coupling degree is low, they destroyed the ecological environment when they were developing [6]. Pei-chao Dai et al. (2004) calculated the coupling degree of Xuzhou with the model of urbanization and ecological environment coupling. It was concluded that Xuzhou entered a state of high coupling from 2001 onwards, and urbanization and ecological environment coordinated development [7]. Bo Li (2015) researched coupled spatiotemporal differences of 29 prefecture level cities of 5 central provinces by using a coupled model of the city, and concluded that the level of urbanization and the ecological environment quality of the coupling development degree in space showed obvious local spatial autocorrelation clustering pattern. The overall trend shows that the in the degree of southeast is higher than that in the northwest [8].

Based on the above literatures, scholars at home and abroad use the coupled model to study the coupling degree of urbanization and ecological environment in different places, but there is no comparative research between the coupling degree of Guizhou Province and other provinces. This paper selects the eastern province of Zhejiang, the central province of Hunan, Guizhou province and Yunnan Province located in the Yunnan Guizhou Plateau. The author collects the data of urbanization and ecological environment, and uses the coupling degree model to calculate the coupling degree of urbanization and ecological environment, and makes a comparative analysis of the four provinces.

\section{Indicators selection and data sources}

The data of this paper is divided into two systems, which are urbanization system and ecological environment system. Urbanization system includes population urbanization subsystem, spatial urbanization subsystem, Economic Urbanization subsystem and social urbanization subsystem. Among them, the population urbanization subsystem includes the proportion of urban population, the proportion of non-agricultural population and percentage of employment in the Tertiary Industry. System space towns including urban area (square kilometers), city population density (per km2), and per capita City Road area (square meters). The sub-system of urbanization includes the per capita disposable income of urban residents (yuan), the proportion of added value of the tertiary industry to GDP and the per capita revenue (yuan). The social urbanization sub-system includes the total retail sales per capita (ten thousand yuan), the number of buses owned by ten thousand people (vehicles), the proportion of the number of Internet users, and the proportion of college students. Ecological environment system includes ecological environment level subsystem, ecological environment pressure subsystem and ecological environment protection subsystem. Among them, the ecological environment subsystem includes water supply per capita (ton/person), city gas penetration rate, per capita green area (m2/person), built-up area green coverage. The pressure of ecological environment subsystem including industrial wastewater emissions per capita (ton/person), per capita amount of industrial solid wastes (ton/person). The ecological environment protection subsystem includes investment in per capita industrial pollution control (yuan), investment in per capita wastewater treatment project (yuan), and investment in per capita pollution control project (yuan).

The data used in this paper are taken from the National Bureau of statistics, as well as the statistical yearbook of the provinces in 2015.

\section{Empirical analysis}

\subsection{Original data standardization}

The original data has positive and negative differences. For example, ecological environment, the index of the ecological environment of pressure subsystems for the reverse index, the higher the index is indicate that the worse the environment is. The positive indicator is in accordance with the formula: $X=\left(x-x_{\max }\right) \div\left(x_{\max }-x_{\min }\right)$. Inverse index is according to the formula: $X=\left(x_{\max }-x\right) \div\left(x_{\max }-x_{\min }\right)$. The raw data are processed according to the formula. $x_{\max }$ is the maximum value of the data in the index. $x_{\min }$ is the minimum value of the data. Urbanization indicators $X$ is defined as $X_{i}(\mathrm{i}=1,2,3 \ldots 13)$. Ecological environment indicators $X$ is defined as $X_{j}(\mathrm{j}=1,2,3 \ldots .11)$.

\subsection{Coefficient of variation and weight of each index}

In this paper, we use the coefficient of variation method to determine the index weight. Variation coefficient method can be better to reflect the possession of weight among all the indicators, the formula is as follows:

$$
\begin{aligned}
& V_{i}=\sigma_{i} / \overline{X_{i}} \\
& V_{j}=\sigma_{j} / \overline{X_{j}}
\end{aligned}
$$


The weights of each index are obtained from the subsystem of urbanization and ecological environment, and the formula is as follows:

$$
\alpha_{\mathrm{i}}=V_{i} \div \sum_{i=1}^{13} V_{i}
$$

Note: $i \mathrm{i}$ is for the urbanization of 13 subsystems $V_{i}$ value.

$$
\beta_{j}=V_{j} \div \sum_{j=0}^{11} V_{j}
$$

Note: $j$ is for the ecological environment of the 11 subsystem $V_{j}$ value. $\alpha$ is the weight of each indicator of the urbanization subsystem, and $\beta$ is the weight of each index of the eco-environment subsystem.

\subsection{Coupling}

Calculate the score of the urbanization system using the following formula.

$$
F=\sum_{i=1}^{13} \alpha_{i} X_{i}, \sum_{i=1}^{13} \alpha_{i}=1
$$

$\alpha$ is every index in the urbanization subsystem. $X_{i}$ is the data in the process of Urbanization.

Calculation of ecological environment subsystem score using the following formula.

$$
G=\sum_{j=1}^{11} \beta_{j} X_{j} \sum_{j=1}^{11} \beta_{j}=1
$$

$\beta$ is the weight of each index in the eco-environment subsystem. $X_{j}$ is the value of the ecological index after treatment.

Calculate the urbanization subsystem score $F$ and the eco-environment subsystem score $\mathrm{G}$ of the four provinces, substitute the coupling degree formula and couple the two systems. Couple the two systems and calculate the coupling degree.

Coupling degree formula:

$$
C=2 \times \frac{\sqrt{(F \times G)}}{F+G}
$$

$F$ value, $\mathrm{G}$ value and the coupling degree of the four provincial capitals are shown in Table 1.

Table 1. Coupling degree.

\begin{tabular}{|c|c|c|c|c|}
\hline & Guizhou & Yunnan & Zhejiang & Hunan \\
\hline F & 0.1928235 & 0.1277376 & 0.802272 & 0.322798 \\
\hline G & 0.2763830 & 0.2646786 & 0.850411 & 0.33653 \\
\hline coupling degree & 0.9840148 & 0.9371344 & 0.999576 & 0.999783 \\
\hline
\end{tabular}

From Table 1, we can see the coupling degree above four provinces are relatively high, which are belong to the highly coupling state. The coupling of Zhejiang and Hunan are close to 1 . The coupling degree of Guizhou and Yunnan are slightly behind, which are higher than 0.9 . The data indicate that the coupling degree between the two systems of the four provinces are in high state, and the urbanization subsystem and the ecological environment subsystem are in the mutual influence.

\subsection{Coupling coordination degree}

Coupling degree can only explain the interaction between the two systems, and the level of urbanization and the coordinated development of the ecological environment can not be explained. So the coupling coordination degree model is introduced to explain this question.

Formula of Coupling Coordination Degree is as the following:

$$
D=\sqrt{C \times T}, T=\alpha F+\beta G
$$

In the formula, $\alpha$ and $\beta$ are the contribution coefficient for the two systems in the development of the city. Here we think that the urbanization subsystem and ecological environment subsystem of urban development is equally important. We set value $\alpha=\beta=0.5$. $D$ is the coupling coordination degree. $C$ is theCoupling degree. The coupling coordination degree is shown in Table 2 
Table 2. Coupling coordination degree.

\begin{tabular}{|l|c|c|c|c|}
\hline & Guizhou & Yunnan & Zhejiang & Hunan \\
\hline Coupling coordination degree & 0.480472 & 0.428805 & 0.908841 & 0.574102 \\
\hline
\end{tabular}

The coupling coordination degree level and the division standard can be seen in Table 3

Table 3. The coupling coordination degree level and the division standard.

\begin{tabular}{|c|c|c|c|}
\hline $\mathrm{D}$ & Degree of coordination & $\mathrm{F}>\mathrm{G}$ & $\mathrm{F}<\mathrm{G}$ \\
\hline $0.8<\mathrm{D} \leq 1$ & Good coordination & The ecological environment lag & Urbanization lag \\
\hline $0.6<\mathrm{D} \leq 0.8$ & Moderate coordination & The ecological environment lag & Urbanization lag \\
\hline $0.4<\mathrm{D} \leq 0.6$ & Low coordination & The ecological environment lag & Urbanization lag \\
\hline $0.2<\mathrm{D} \leq 0.4$ & Moderate disorder & The ecological environment lag & Urbanization lag \\
\hline $0<\mathrm{D} \leq 0.2$ & Severe disorder & The ecological environment lag & Urbanization lag \\
\hline
\end{tabular}

From Table 3 we can find out that Guizhou province is in a level of low imbalance and urbanization hysteresis. The governments of Guizhou province care both the development of economy and the environmental protection, but the coordination degree between the two systems is not high. The $F$ value of urbanization subsystem is 0.1928 , which is much less than that of Zhejiang (0.8023), and 0.3228 of Hunan Province. Therefore, there is still room for the improvement of the urbanization of Guizhou province. The Urbanization subsystem and ecological environment subsystem has a large gap in Guizhou province, which means Guizhou province not only focused on the development of urbanization but also paid attention to the protection of the ecological environment, and the government takes the road of sustainable development. Yunnan province is in the level of low coordination and urbanization hysteresis, and the urbanization level in Yunnan is much lower than the ecological environment subsystem. This can be due to its main industry (tourism industry). Yunnan Province has paid great attention to the protection of the environment, and because the development of tourism industry is quite good, the villages have some functions of cities and towns, thus limiting the urbanization of Yunnan Province to a certain extent. Zhejiang Province, as the eastern economic strong province of China, is in the level of high coordination and Zhejiang's urbanization index is far ahead of the other two western provinces and Hunan Province. And because the economy is well developed, the investment in ecological environment is also increased, therefore the ecological environment subsystem value in Zhejiang province is also in high level. Urbanization drives the development of economy, while economic development is also promoting the construction of ecological environment. Hunan is in the level of low imbalance, and its urbanization is almost keep pace with the ecological environment. As a province with a certain economic base in central China, Hunan Province has developed rapidly and has a relatively high urbanization system. Hunan Province also invest a certain amount for the protection of ecological environment. But there are multiple economic indicators are relatively left behind, and its per capita investment funds in environmental protection is less than that of the two western provinces, so there are a lot of room for the improvement of urbanization and ecological environment protection investment in Hunan province.

\section{Conclusions and recommendations}

In this paper, the coupling degree model is used to analyze the coupling development of urbanization and ecological environment in Guizhou Province, Yunnan Province, Hunan Province and Zhejiang province. The results of the comparative analysis show that the coupling degree of Guizhou province is highly coupled, which is as high as 0.9840 . Among these four provinces, the coupling degree of Guizhou province occupies the third position, which is only 0.4805 , and the gap between Hunan province and Zhejiang province is not very large. The index of urbanization subsystems lower than the index of ecosystem in Guizhou province. The coupling degree and coupling coordination degree of Zhejiang province are both high, and they are highly coupled and in good coordination. The coupling degree of Hunan province is highly coupled, and the coupling coordination degree is in the level of low coordination. The coupling degree of Yunnan province is highly coupled, and the coupling coordination degree is inthe level of low coordination. According to the research results of this paper, here we present the following policy recommendations:

Firstly, Guizhou provincial government should vigorously promote urbanization and strengthen the construction of ecological environment. Under the trend of the national vigorously promoting urbanization, Guizhou province should grasp the opportunity to promote urbanization and enjoy the benefits of economic development. At the same time, the 
government should strengthen the construction of ecological environment, promote the construction of urban green space, increase the investment of environmental protection, and vigorously promote the tourism industry and the development of large data industry, in order to promote the harmonious development of urbanization and ecological environment.

Secondly, the tourism industry of Yunnan province helps the development of small towns and rural economy. In the future, Yunnan province should continue to put ecological environment protection as the first priority, and continue to promote the construction of tourist attractions around the urbanization. Zhejiang province should vigorously develop green economy, complete economic transformation, and further promote the development of urbanization and ecological environment. As a population outflow province, Hunan province should formulate policies to promote the older generation of migrant workers to settle in towns and enjoy the welfare of social development. In the construction of ecological balance, Hunan province is a developed province in the steel making industry and chemical industry. In the past, the ecological environment of Hunan province has been polluted seriously. Therefore, Hunan province should pay attention to the experience, and push on the coordinated development of ecological balance and urbanization.

\section{References}

1. People's Publishing House. National new urbanization planning, People's Publishing House, (2014).

2. Z.G. Sun, The people's government of Guizhou province in 2016, "the government work report". Guizhou daily, (2016).

3. G.M. Grossman, A.B. Krueger, Economic growth and the environment. Q J. Econ, 110(2):353-377, (1995).

4. G. Haughton, C. Hunter, Sustainable cities, London: Jessica Kingsley Publishers Ltd, (1994).

5. Y.L. Xiao, Study on the coupling development model of urbanization and ecological environment in Guizhou. Guiyang: Guizhou University of Finance and economics, (2013).

6. N.S. Luo, J.J. Li, Fu, F.Z. Luo, The coupling relationship between urbanization and ecological environment - A case study of Changsha, Zhuzhou, Xiangtan city group. Hunan forum, 27, (2014).

7. P.C. Dai, Z.P. Shen, Y.L. Zhao et al, Changes in coupling relationship between urbanization and ecological environment: a case of Xuzhou City, Journal of Jiangsu Normal University (Natural Science Edition), 32, (2014).

8. B. Li, J.X. Zhang, The regional differences and temporal and spatial evolution on urbanization and ecological environment in the central plains economic region, Regional Research and Development, 34, (2015). 